\title{
How the First Stars Regulated Star Formation. II. Enrichment by Nearby Supernovae
}

\author{
Ke-Jung Chen ${ }^{1,2,3,7}$, Daniel J. Whalen ${ }^{4}$, Katharina M. J. Wollenberg ${ }^{5}$, Simon C. O. Glover ${ }^{5}$, and Ralf S. Klessen ${ }^{5,6}$ \\ ${ }^{1}$ Division of Theoretical Astronomy, National Astronomical Observatory of Japan, Tokyo 181-8588, Japan; ken.chen@ nao.ac.jp \\ ${ }^{2}$ Institute of Astronomy and Astrophysics, Academia Sinica, Taipei 10617, Taiwan \\ ${ }^{3}$ Department of Astronomy \& Astrophysics, University of California, Santa Cruz, CA 95064, USA \\ ${ }^{4}$ Institute of Cosmology and Gravitation, Portsmouth University, Portsmouth, UK \\ ${ }^{5}$ Zentrum für Astronomie, Institut für Theoretische Astrophysik, Universität Heidelberg, Germany \\ ${ }^{6}$ Interdisziplinäres Zentrum für Wissenschaftliches Rechnen, Universität Heidelberg, Germany \\ Received 2016 September 29; revised 2017 June 14; accepted 2017 June 20; published 2017 July 27
}

\begin{abstract}
Metals from Population III (Pop III) supernovae led to the formation of less massive Pop II stars in the early universe, altering the course of evolution of primeval galaxies and cosmological reionization. There are a variety of scenarios in which heavy elements from the first supernovae were taken up into second-generation stars, but cosmological simulations only model them on the largest scales. We present small-scale, high-resolution simulations of the chemical enrichment of a primordial halo by a nearby supernova after partial evaporation by the progenitor star. We find that ejecta from the explosion crash into and mix violently with ablative flows driven off the halo by the star, creating dense, enriched clumps capable of collapsing into Pop II stars. Metals may mix less efficiently with the partially exposed core of the halo, so it might form either Pop III or Pop II stars. Both Pop II and III stars may thus form after the collision if the ejecta do not strip all the gas from the halo. The partial evaporation of the halo prior to the explosion is crucial to its later enrichment by the supernova.
\end{abstract}

Key words: cosmology: theory - early universe - galaxies: high-redshift - intergalactic medium stars: Population III - supernovae: general

\section{Introduction}

How metals from the first supernovae $(\mathrm{SNe})$ are taken up into new stars governs the transition from Population III (Pop III) to Population II (Pop II) star formation in the primordial universe (Bromm et al. 2001; Schneider et al. 2002; Mackey et al. 2003; Smith \& Sigurdsson 2007; Clark et al. 2008; Jappsen et al. 2009; Smith et al. 2009; Dopcke et al. 2011; Ritter et al. 2012, 2016; Chiaki et al. 2013; Safranek-Shrader et al. 2014; Chen et al. 2015). This transition in turn regulates the luminosities, spectra, and star formation rates of primeval galaxies (Greif et al. 2008, 2010; Johnson et al. 2009; Pawlik et al. 2011, 2013; Jeon et al. 2012; Wise et al. 2012; O'Shea et al. 2015), the demographics and rates of supermassive black hole formation (Alvarez et al. 2009; Tanaka \& Haiman 2009; Park \& Ricotti 2011; Agarwal et al. 2012; Park \& Ricotti 2012; Volonteri 2012; Whalen \& Fryer 2012; Choi et al. 2013; Latif et al. 2013a, 2013b; Park \& Ricotti 2013; Schleicher et al. 2013; Johnson et al. 2014; Haemmerlé et al. 2017; Smidt et al. 2017; Woods et al. 2017), and the onset of cosmological reionization (Kitayama et al. 2004; Whalen et al. 2004; Alvarez et al. 2006; Abel et al. 2007). This transition also influences the rate at which primordial SNe may be detected in future surveys (Whalen et al. 2008b, 2013a, 2013b, 2013c, 2013d, 2013e, 2013f, 2014a, 2014b; Kasen et al. 2011; Mesler et al. 2012, 2014; de Souza et al. 2013, 2014; Chen et al. 2014a, 2014b, 2014c; Smidt et al. 2014, 2015; Magg et al. 2016) and the number of low-mass Pop III stars and secondgeneration stars expected in Galactic archaeological searches (Beers \& Christlieb 2005; Frebel et al. 2005; Joggerst \& Whalen 2011; Hartwig et al. 2015).

Cosmological simulations usually take this transition to occur when metals from Pop III SNe touch a halo because they

EACOA Fellow. cannot resolve the actual interaction of the metals with the gas in the halo. They may therefore overestimate the true mixing efficiency and predict a premature transition from Pop III to Pop II star formation. Past, higher-resolution simulations seem to indicate that metals from $\mathrm{SNe}$ in reality do not mix with nearby halos very efficiently. Cen \& Riquelme (2008) modeled SN shocks colliding with $10^{6}-10^{7} M_{\odot}$ halos at $10-100 \mathrm{~km} \mathrm{~s}^{-1}$ at $z \sim 10$. They found that mild shear flows driven by KelvinHelmholtz (KH) instabilities limited mixing to the outer regions of the halo, and that at most one-third of the baryons at high densities in the halo were enriched above $3 \%$ of the metallicity of the ambient intergalactic medium (IGM) by $z \sim 6$. They concluded that the Pop III/Pop II transition may therefore have been protracted, with Pop III stars forming at lower redshifts than previously thought. More recent studies indicate that metals can reach greater depths in the gas if it is turbulent, although some rely on ad hoc subgrid models for turbulent mixing (Gray \& Scannapieco 2011; Richardson et al. 2013).

But these models do not include the photoevaporation of the halo by the star prior to its explosion. Studies have shown that ionizing UV radiation from nearby Pop III stars can ablate the outer layers of the halo in supersonic flows and expose its interior to the IGM (Shapiro et al. 2004; Iliev et al. 2005; O'Shea et al. 2005; Susa \& Umemura 2006; Susa 2007; Whalen et al. 2008a, 2010; Hasegawa et al. 2009; Susa et al. 2009). The dense core casts a cylindrical shadow that is eventually crushed down into the axis of the halo by thermal pressure forces in the surrounding ionized gas. If metals from an SN collide with the ablative flow, they can mix violently with it and cool it, perhaps causing it to fragment and collapse into new stars. The metals can also envelop the partially exposed core, enriching and cooling it to greater depths than those found by Cen \& Riquelme (2008). The collision of SN ejecta with neighboring halos may therefore trigger new star formation, not just enrich the halo. 
How metals mix with halos in such scenarios is also crucial to predicting elemental abundances in the second-generation stars that do form, some of which may still exist today as ancient, dim metal-poor stars in the Galactic halo (e.g., Cayrel et al. 2004; Lai et al. 2008; Frebel 2010; Caffau et al. 2013; Norris et al. 2013; Aoki et al. 2015; Bonifacio et al. 2015). If the abundances found in extremely metal-poor stars can be reconciled to the nuclear yields of Pop III SNe, they can place constraints on the masses of the first stars (Iwamoto et al. 2005; Cooke et al. 2011, 2015; Caffau et al. 2012; Cooke \& Madau 2014). So far, the fossil abundance record suggests that early chemical enrichment is due more to core-collapse (CC) than massive pair-instability (PI) SNe (Joggerst et al. 2010; but see Karlsson et al. 2008; Aoki et al. 2014). Past studies have focused just on the yields, not the dilution factors with which metals are found in stars. Numerical simulations that resolve both mixing and cooling in pristine gas are needed to reproduce these concentrations in specific stars, such as J031300 (Keller et al. 2014; Chen et al. 2017).

Here we examine the collision of ejecta from Pop III SNe with nearby halos as a potential channel of prompt second-generation star formation in the early universe with the ZEUS-MP and CASTRO codes. Our simulations include the evaporation of the halo by the star prior to the explosion and resolve mixing on spatial scales that are not currently practical in cosmological simulations. In Section 2 we describe our halo evaporation and SN blast models, which were done with ZEUS-MP. In Section 3, we present the mixing calculations with CASTRO and examine how metals from CC and PI SNe mix with halos with a variety of masses and evaporation states. We discuss the prospects for second-generation star formation after the collision and conclude in Section 4.

\section{Numerical Method}

Our simulations are carried out in two stages. First, the halo is photoionized by the external star with the ZEUS-MP cosmological radiation transport code. The collision of ejecta from the explosion of this star with the ionized halo is then modeled with CASTRO. In CASTRO, the SN ejecta is treated as a time-dependent, plane-wave inflow at the lower boundary of the simulation box that is extracted from 1D expanding grid models of the SN in ZEUS-MP. We model mixing in two stages because ZEUS-MP has the radiation transport required to evaporate the halo and CASTRO has the adaptive mesh refinement $(\mathrm{AMR})$ required to resolve the collision of the ejecta with the halo.

\subsection{ZEUS-MP}

ZEUS-MP is a massively parallel radiation hydrodynamics code that evolves astrophysical fluid flows self-consistently with nonequilibrium nine-species primordial gas chemistry and multifrequency photon-conserving ray-tracing UV transport to evolve cosmological ionization fronts (I-fronts; Whalen \& Norman 2006, 2008a, 2008b). The halo photoevaporation models in this study are identical in physics to those in Whalen et al. (2008a, 2010); here, we review the properties of the stars, the halos, and the simulation box in ZEUS-MP.

\subsubsection{Halo Models}

We consider 25 and $200 M_{\odot}$ stars at a distance of $250 \mathrm{pc}$ to $6.9 \times 10^{5}, 2.1 \times 10^{6}$, and $1.2 \times 10^{7} M_{\odot}$ halos. These halos

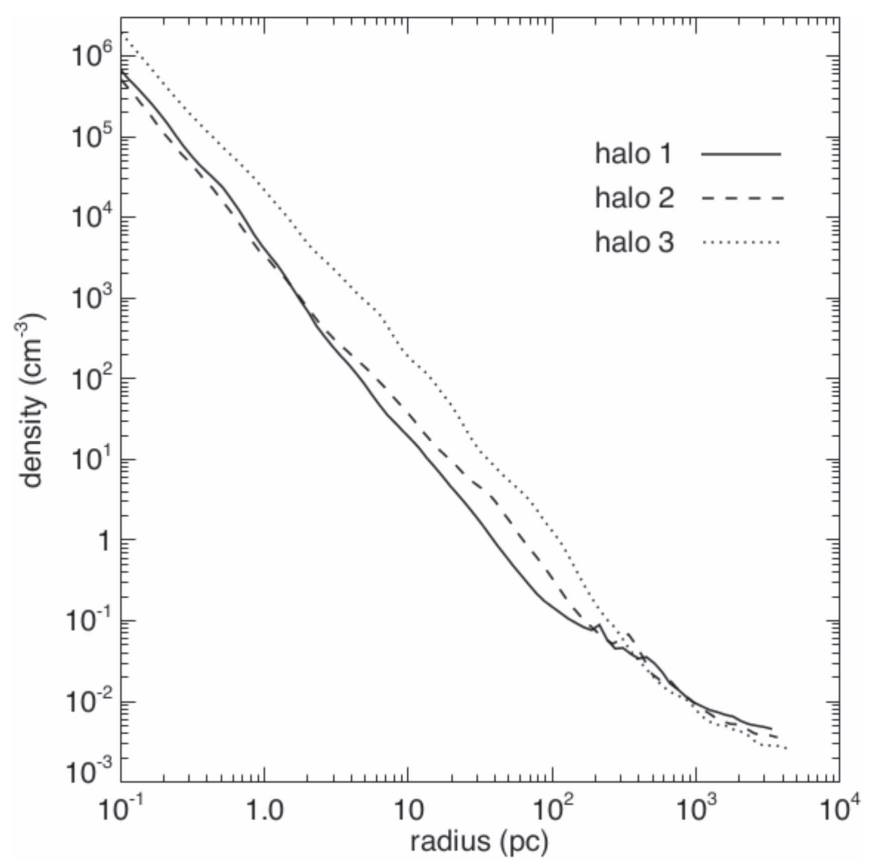

Figure 1. Spherically averaged density profiles for the $6.9 \times 10^{5} M_{\odot}$ (halo 1), $2.1 \times 10^{6} M_{\odot}$ (halo 2), and $1.2 \times 10^{7} M_{\odot}$ (halo 3) models.

bracket the range in mass in which Pop III stars form via $\mathrm{H}_{2}$ cooling and are extracted from cosmological simulations performed with the Enzo code (Bryan et al. 2014). We show spherically averaged density profiles of all three halos prior to illumination in Figure 1. As seen from the central densities, they are at intermediate stages of collapse but have not yet formed stars. The densities, gas energies, velocities, and species mass fractions of these halos are spherically averaged and mapped onto a two-dimensional (2D) cylindrical coordinate grid in ZEUS-MP. The ionizing UV fluxes and lifetimes of the two stars are taken from Schaerer (2002). Each halo is centered on the $z$-axis, with only its upper hemisphere residing on the grid. The grid has 1000 zones in $z$ and 500 zones in $r$ for a spatial resolution of $0.25 \mathrm{pc}$. The boundaries are at $-125 \mathrm{pc}$ and $125 \mathrm{pc}$ in $z$ and $0.01 \mathrm{pc}$ and $125 \mathrm{pc}$ in $r$. Reflecting boundary conditions (BCs) are applied to the inner $r$ boundary, and outflow BCs are set on the other three boundaries. All simulations were performed at redshift $z=20$.

\subsubsection{Photoevaporated Halos}

We show $\mathrm{H}_{2}$ mass fractions for the partially evaporated halo when the star dies in Figure 2 (the process of halo photoevaporation is discussed in greater detail in Whalen et al. 2008a, 2010). The D-type I-front has a cometary appearance because it preferentially advances in the more stratified densities above and below the midplane of the halo. A prominent arc of $\mathrm{H}_{2}$ is visible in the outer layers of the I-front. $\mathrm{H}_{2}$ forms there because the hard UV spectrum of the star broadens the I-front, creating temperatures of a few thousand kelvin and ionization fractions of $\sim 0.1$ in its outer layers that catalyze the rapid formation of $\mathrm{H}_{2}$ via the $\mathrm{H}^{-}$channel (Ricotti et al. 2001). The $\mathrm{H}_{2}$ in the outer layers of the I-front partially shields the core from Lyman-Werner (LW) photons from the star, as shown by the large $\mathrm{H}_{2}$ mass fractions there. The core also shields gas behind it from LW flux. 


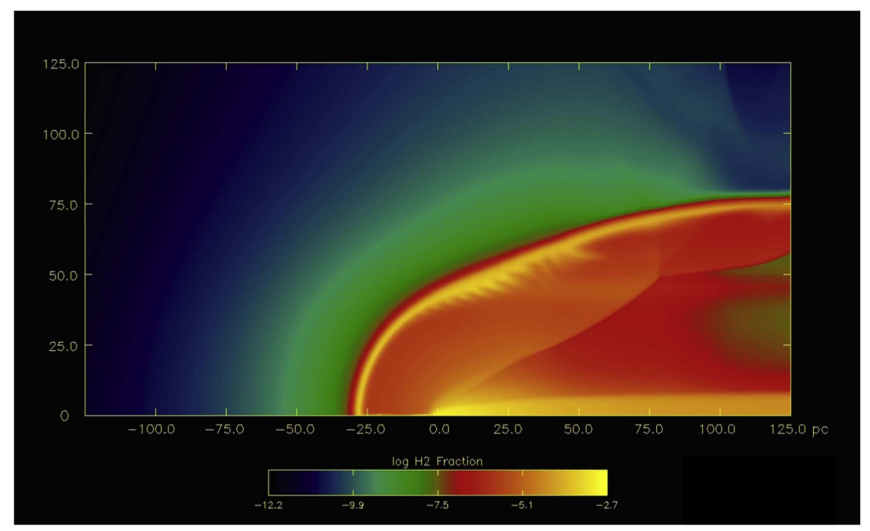

Figure 2. $\mathrm{H}_{2}$ mass fractions for the partially evaporated $6.9 \times 10^{5} M_{\odot}$ halo that is $250 \mathrm{pc}$ from the $25 M_{\odot}$ star at the end of the life of the star. The horizontal and vertical axes are $z$ and $r$, respectively.

The serrated structures midway up the arc of the front are remnants of dynamical instabilities that are driven by $\mathrm{H}_{2}$ cooling in the outer layers of the front. They are more prominent farther out along the arc because of the larger angles of incidence between the photons and the front there (Williams 2002). These structures are of interest because they enhance mixing between outflows from the halo and the SN ejecta. Supersonic flows are also driven back toward the star as the outer layers of the halo are blown off by the radiation front. These flows collide with and mix with ejecta from the SN from the left, as discussed later.

After the star dies, the halo and $\mathrm{HII}$ region are evolved in ZEUS-MP for the additional time required for metals from the $\mathrm{SN}$ to reach the left boundary of the grid in CASTRO. The pressure of the ionized gas continues to crush the shadow cast by the halo downward toward its axis after the explosion, and the supersonic backflows keep expanding toward the ejecta. The ionized gas also begins to recombine out of equilibrium, cooling more quickly than it recombines. Following these three processes for the time it takes debris from the $\mathrm{SN}$ to reach the boundary of the CASTRO box ensures that the metals encounter a more realistic circumhalo structure at the time of collision. The time required for the ejecta to cross this distance is taken from the 1D ZEUS-MP SN models described below. The halos are illuminated by the $25 M_{\odot}$ star for its main-sequence lifetime of $6.46 \mathrm{Myr}$, after which radiation is switched off, and the $\mathrm{H}$ II region is allowed to cool and recombine for an additional $2.16 \mathrm{Myr}$. For the $200 M_{\odot}$ star these two times are 2.2 and $0.872 \mathrm{Myr}$, respectively.

\subsection{CASTRO}

The halo and surrounding $\mathrm{H}$ II region are mapped onto a 2D Cartesian grid in CASTRO with a bilinear interpolation scheme. Since only the upper half of the halo is modeled in ZEUS-MP, its lower half is assumed to be the mirror image of its upper half. CASTRO is a multidimensional AMR radiation hydrodynamics code (Almgren et al. 2010; Zhang et al. 2011) with an unsplit piecewise parabolic hydro scheme (Woodward \& Colella 1984) and an ideal gas equation of state with $\gamma=5 / 3$. We advect three species (hydrogen, helium, and metals) and use a simple cooling function from Gnedin \& Hollon (2012) but neglect the detailed primordial gas chemistry and cooling and metal line cooling. The mass fractions of the five $\mathrm{H}$ species in the ZEUS-MP models are summed to obtain the single $\mathrm{H}$
Table 1

Target Halos and SNe

\begin{tabular}{lccl}
\hline \hline Model & $\begin{array}{c}\text { Halo Mass } \\
\left(M_{\odot}\right)\end{array}$ & $\begin{array}{c}R_{200} \\
(\mathrm{pc})\end{array}$ & SN Type \\
\hline h1sn25 & $6.9 \times 10^{5}$ & 249.6 & CC \\
h1sn200 & $6.9 \times 10^{5}$ & 249.6 & PI \\
h2sn25 & $2.1 \times 10^{6}$ & 320.1 & CC \\
h2sn200 & $2.1 \times 10^{6}$ & 320.1 & PI \\
h3sn25 & $1.2 \times 10^{7}$ & 483.1 & CC \\
h3sn200 & $1.2 \times 10^{7}$ & 483.1 & PI \\
\hline
\end{tabular}

Note. $R_{200}=R\left(\rho=200 \Omega_{b, 0} \rho_{\text {crit }}\right)$.

species in CASTRO, and the three He species are summed to obtain the one He species. The coarse grid is $250 \mathrm{pc}$ in $x$ and $y$ with $1024^{2}$ zones. Up to four levels of refinement are allowed for a factor of $16\left(2^{4}\right)$ higher spatial resolution. Refinements are performed on gradients in density and velocity.

Four nested grids are centered on the halo for a maximum resolution of $0.015 \mathrm{pc}$, or $3000 \mathrm{au}$. Inflow BCs are set on the lower $y$ boundary, where SN ejecta flow onto the grid as described below, and outflow BCs are set on the other three boundaries so metals and ionized gas can exit the grid. The two explosions, the $25 M_{\odot} \mathrm{CC} \mathrm{SN}$ and the $200 M_{\odot}$ PI SN, have energies of 1 and $42 \mathrm{~B}$, respectively ( $1 \mathrm{~B}=10^{51} \mathrm{erg}$ ). The halo and metals are evolved for $20 \mathrm{Myr}$. We summarize our models in Table 1. These 2D simulations required 480,000 CPU hours on the Cray XC30 machines at the National Energy Research Scientific Computing Center (NERSC) and the Center for Computational Astrophysics (CfCA) at the National Astronomical Observatory of Japan (NAOJ).

\subsubsection{SN Inflow Model}

We treat the $\mathrm{SN}$ ejecta as a time-dependent planar inflow on the lower $y$ boundary. Gas densities, energies, velocities, and mass fractions for all three species are updated on the boundary every time step during the simulation. These time-dependent flows are derived from 1D explosion models of the 25 and $200 M_{\odot}$ SNe in ZEUS-MP. In these 1D models, the SN is evolved in its own halo as in Whalen et al. (2008c), but only out to radii corresponding to the position of the lower boundary of the box in CASTRO. These simulations are done in two steps. First, the H II region of the star in its own halo is created as described in Whalen et al. (2008c) and Schauer et al. (2015, 2017), with ionizing photon rates again taken from Schaerer (2002) for consistency. The host halos of the 25 and $200 M_{\odot}$ stars are taken to be identical to the $6.9 \times 10^{5}$ and $2.1 \times 10^{6}$ $M_{\odot}$ neighbor halos, respectively. This choice of host halos is arbitrary, but reasonable. Both stars ionize their own halos within a few hundred kiloyears, with supersonic flows from the core plowing most of the baryons into a dense shell with a radius of $100-200 \mathrm{pc}$ by the time the star dies. At this point the densities at the center of the halo are low, $\sim 0.1-1 \mathrm{~cm}^{-3}$ (e.g., Figure 3 of Whalen et al. 2004), so both stars explode in diffuse media.

We then explode the star in the center of its $\mathrm{H}$ II region on a 1D expanding grid in ZEUS-MP as explained in Whalen et al. (2008c; see also Meiksin \& Whalen 2013). The blast wave is initialized as a free expansion with density and velocity profiles from Truelove \& McKee (1999), which are parameterized by ejecta mass, $M_{\mathrm{ej}}$, explosion energy, $E_{\mathrm{ej}}$, and peak ejecta 

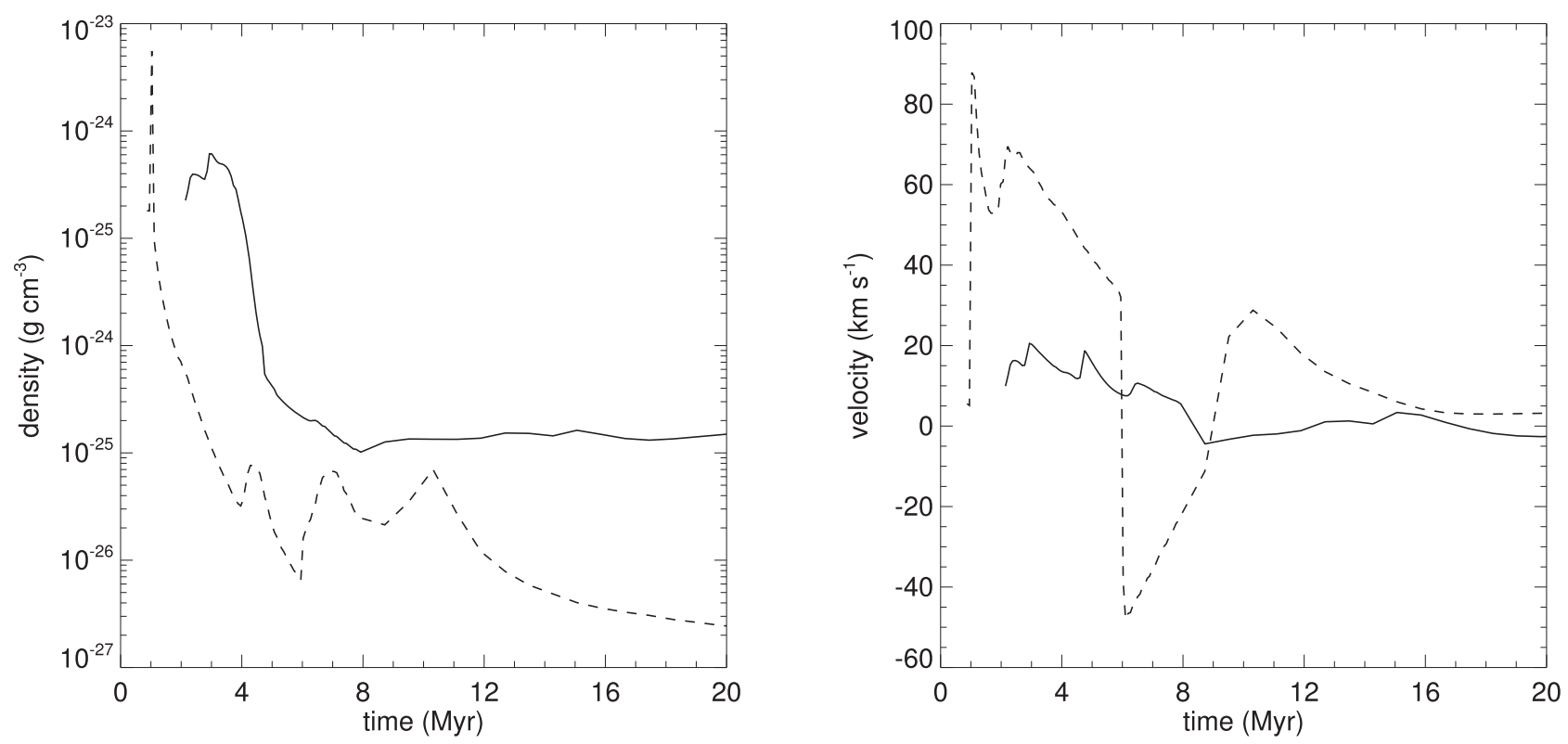

Figure 3. Time-dependent boundary conditions representing the flow of ejecta from a Pop III SN past a fixed observer position at $125 \mathrm{pc}$, derived from $1 \mathrm{D}$ expanding grid explosion models done with ZEUS-MP. Both explosions occur 250 pc from the halo. Left panel: densities. Dashed line: the $200 M_{\odot}$ PI SN; solid line: the $25 M_{\odot}$ CC SN. Right panel: velocities. Dashed line: the $200 M_{\odot}$ PI SN; solid line: the $25 M_{\odot}$ CC SN.

velocity, $v_{\text {max }}$. We take $M_{\text {ej }}=20 M_{\odot}$ and $E_{\text {ej }}=1 \mathrm{~B}$ for the $25 M_{\odot}$ SN (assuming the formation of a $5 M_{\odot}$ black hole during the core collapse) and $M_{\mathrm{ej}}=200 M_{\odot}$ and $E_{\mathrm{ej}}=42 \mathrm{~B}$ for the $200 M_{\odot}$ star (no compact remnant; Heger \& Woosley 2002). In both cases $v_{\max }=3.0 \times 10^{9} \mathrm{~cm} \mathrm{~s}^{-1}$. The profiles were initialized on a 1D spherical grid with 250 uniform zones and an outer boundary at $4.626 \times 10^{15} \mathrm{~cm}$. These models are otherwise identical in physics to those in Whalen et al. (2008c) and Meiksin \& Whalen (2013), in which the importance of inverse Compton cooling to the evolution of Pop III SN remnants in the primordial universe is reviewed in detail (see also Kitayama \& Yoshida 2005).

The SN remains essentially a free expansion until it has swept up about its own mass in ambient gas at radii of $10-20 \mathrm{pc}$. At this point a reverse shock forms and is driven back toward the center of the halo in the frame of the shock, reverberating back and forth through the ejecta over time in some runs. When the ejecta crash into the dense shell of the relic $\mathrm{H}$ II region, the collision can drive another reverse shock into the interior of the halo. To an observer at a fixed radius (such as at the lower boundary of the CASTRO box), passing flows would therefore be highly time dependent, with strong forward flows at some times and backflow at others.

We show velocities and densities at $125 \mathrm{pc}$, the position of the lower boundary in CASTRO, for the $25 M_{\odot}$ CC SN and $200 M_{\odot}$ PI SN in Figure 3. They are extracted from the 1D ZEUS-MP run over seven or eight decades in time at uniform intervals in log time. The passage of the shock and the rarefied regions in its wake are visible as the peaks and valleys in density. The peak happens earlier in the PI SN flow because it is more energetic and reaches the outer regions of the halo sooner. Both forward and reverse shocks are visible in the velocity profiles, with the latter causing negative velocities in both flows. To compute the SN ejecta entering the CASTRO grid, the densities and velocities shown in Figure 3 (and energy densities and mass fractions) are stored in a table as a function of time. At a given time step in CASTRO the inflow BCs are computed with a linear interpolation of the logarithm of the table entries versus log time.

Our simulations using two different codes with different dimensions and physics are conceptually complicated. Therefore, we use a cartoon to summarize the entire numerical setup in Figure 4 to make it clearer.

\section{Halo Enrichment}

\subsection{Halo Structure at Collision}

We show density and temperature images of the partially evaporated halos and their ionized envelopes at the time the SN enters the lower boundary of the grid in CASTRO in Figures 5 and 6. After the star dies, the ionized gas crushes the shadow cast by the halo down toward its axis, even as it cools and recombines. The solid angle cast by the halo varies with its mass, its proximity to the star, and the luminosity of the star. More massive halos and less luminous stars cast the broadest shadows, while less massive halos and more luminous stars create narrow cocoons with an almost cometary appearance. The dense ridges of gas created by I-front instabilities in Figure 2 are visible in the $\mathrm{h} 1 \mathrm{sn} 25$ profile but do not appear in the other halos. In general, more massive stars with higher fluxes create less $\mathrm{H}_{2}$ in the outer layers of the I-front, so there is less cooling and instability growth. I-fronts from any given star are also less likely to form instabilities in more massive halos because it is more difficult for them to develop in higher densities.

By this time, regions of the halo and its now partially ionized envelope have cooled to $\sim 150 \mathrm{~K}$ because of rapid $\mathrm{H}_{2}$ formation after the death of the star. In the absence of UV flux the ionized gas begins to recombine out of equilibrium, cooling more quickly than it becomes neutral. Denser regions of the gas fall to temperatures and ionized fractions of a few thousand kelvin and $\sim 0.1$ first, triggering rapid $\mathrm{H}_{2}$ formation via the $\mathrm{H}^{-}$ channel. This process is what cools gas in the ionized envelope of the halo. Temperatures in the core and its shadow fall over time even though they were never ionized because they are no 

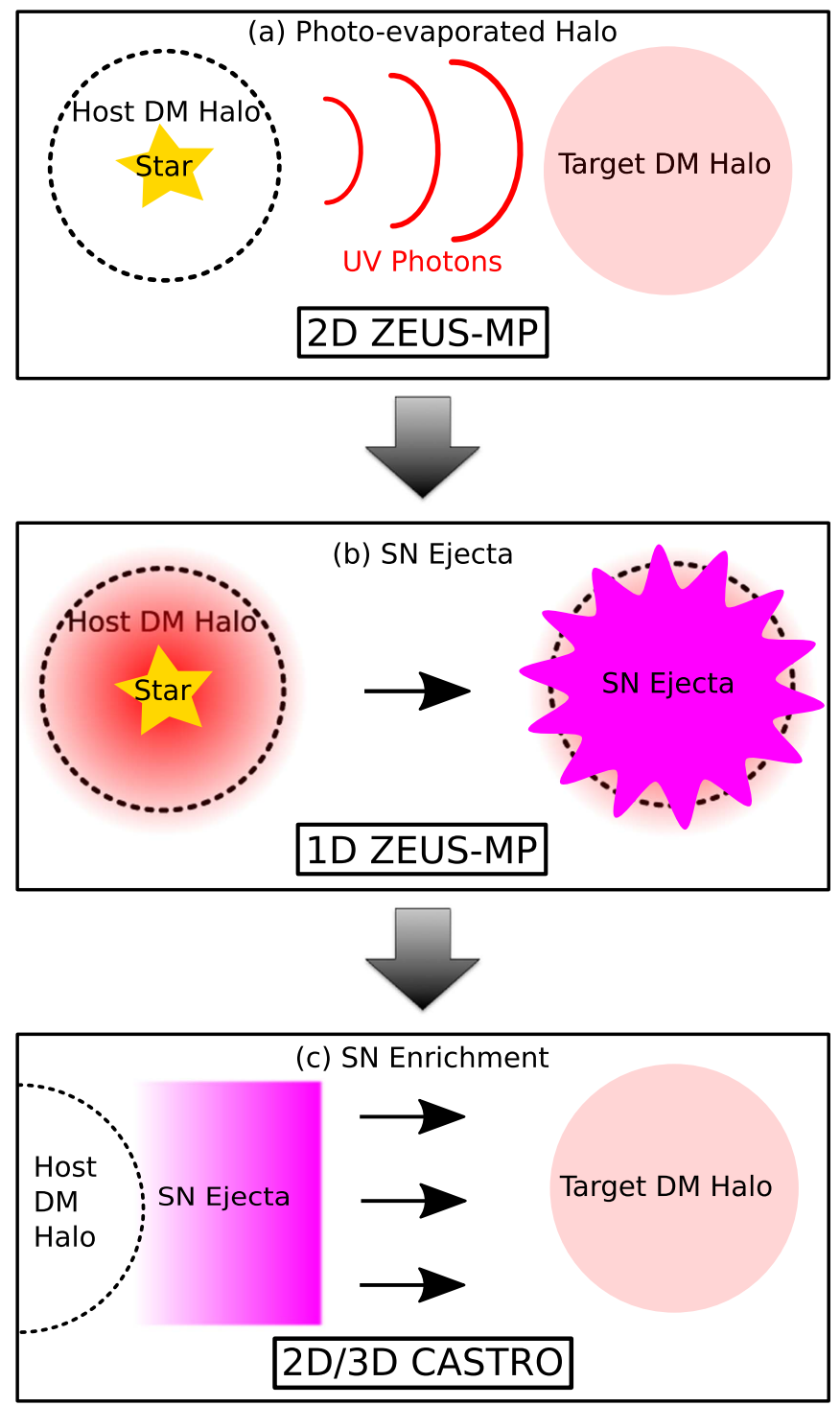

Figure 4. Simulation flowchart. Our numerical setup can be divided into three steps shown in panels (a)-(c). We first use 2D ZEUS-MP to simulate UV feedback to the target halo until the star dies as an SN, and then we simulate the propagation of SN ejecta in 1D ZEUS-MP before they reach the boundary of the CASTRO box. Finally, we use the initial and boundary conditions from two previous steps to simulate the cosmological mixing in multiple dimensions with CASTRO.

longer exposed to LW flux from the star, so $\mathrm{H}_{2}$ rapidly forms in the high densities there and cools the gas. The cold gas in the thin layers tracing the remnant of the I-front is due to $\mathrm{H}_{2}$ that formed there while the star was alive. It is coldest because the $\mathrm{H}_{2}$ is located in the dense gas plowed up by the front. Low gas temperatures in Figure 6 track regions of higher density in Figure 5 because $\mathrm{H}_{2}$ formation is a two-body process.

We include $\mathrm{H}_{2}$ cooling in the relic $\mathrm{H}$ II region because it can enhance mixing during collision with SN ejecta because it condenses the gas to higher densities. Note that had HD chemistry been included in our models, it would have cooled the gas even further, down to the cosmic microwave background $(\mathrm{CMB})$ temperature $(\sim 57 \mathrm{~K}$ at $z=20)$, because the nonsymmetric HD molecule can radiate via dipole transitions while $\mathrm{H}_{2}$ can only emit via quadrupole transitions (e.g., Glover $\&$ Abel 2008). Lower temperatures might have resulted in even higher densities and more efficient mixing. HD cooling is also known to lower the mass scales of fragmentation in relic $\mathrm{H} \mathrm{II}$ regions (see Yoshida et al. 2007a, 2007b), which leads to the formation of Pop III stars that are somewhat lower in mass than those forming from $\mathrm{H}_{2}$ cooling alone.

\subsection{Collision of the $S N$ with the Halo}

We show the collision of the SN with the halo in the h1sn25 and h1sn200 models in the upper panels of Figure 7. Ejecta enter the lower boundary in CASTRO at $\sim 20 \mathrm{~km} \mathrm{~s}^{-1}$ for the CC $\mathrm{SN}$ and $\sim 30 \mathrm{~km} \mathrm{~s}^{-1}$ for the PI SN. As the shock approaches the halo, it decelerates as it collides with the photoevaporative backflow and then climbs up the density gradient of the halo, advancing to within $\sim 100$ pc of its core by $3-5 \mathrm{Myr}$. In the h1sn 25 run the shock stalls 50 pc from the center of the halo. The h1sn200 shock is more energetic and gets to within $5 \mathrm{pc}$ of the center. In both runs the stripping of the outer layers of the halo by ionizing UV photons from the star allows metals to reach greater depths in the halo than would otherwise have been possible. The remnants of the I-front instabilities that are visible in the density image of the h1sn 25 model at $5 \mathrm{Myr}$ develop into an extended turbulent layer as the SN shock overruns the system. This structure dominates the dynamics of the entire envelope at $10 \mathrm{Myr}$ and results in very efficient mixing.

If the shock is strong enough and the densities it encounters are not too high, a reverse shock forms and detaches from the forward shock as it plows up more gas. The contact discontinuity separating the two shocks crumples into Rayleigh-Taylor (RT) instabilities that in turn drive $\mathrm{KH}$ shear instabilities, and the metals begin to mix with pristine gas in the halo. If the shock is weaker or less of the halo is evaporated by the star, the RT instabilities, if they form, are milder and less mixing occurs. Both cases are shown in the lower panels of Figure 7. The stronger PI SN shock drives more mixing than the $\mathrm{CC}$ SN in a given halo. Mixing is weaker in more massive halos for a given SN because they lose less gas to photoevaporation prior to the explosion and present more mass to the shock.

The most mixing occurs in h1sn200, in which metals from the SN reach the core of the halo. In this model only metalenriched Pop II stars would form, in the layers facing the explosion and in its center. Metals do not reach the core in the h2sn200 and h3sn200 runs, so both Pop III and Pop II stars may not form in these models. In contrast, Pop II star formation, if it occurs, would only happen in a narrow arc $50-100 \mathrm{pc}$ in front of the center of the halo in the sn25 runs. The top panel of Figure 7 illustrates that this is the only region that has sufficient metal enrichment. One caveat is the relative timescales of Pop II and Pop III star formation in the h2sn200 and h3sn200 models. If Pop II stars form more quickly in regions with metals than Pop III stars form in the core, LW and $\mathrm{H}^{-}$photodetaching photons from the Pop II stars could destroy $\mathrm{H}_{2}$ in the core, delaying cooling and collapse. Ionizing photons could then later mechanically disrupt the core and prevent its collapse altogether. Improved simulations with metal and dust cooling that are able to resolve fragmentation and collapse will determine the relative timescales of Pop II and III star formation in future models.

On the other hand, if a Pop III star forms in the core of the halo before its outer layers cool, fragment, and collapse to Pop II stars, they may never get the chance to form because UV from the Pop III star breaks out of the halo and completely 

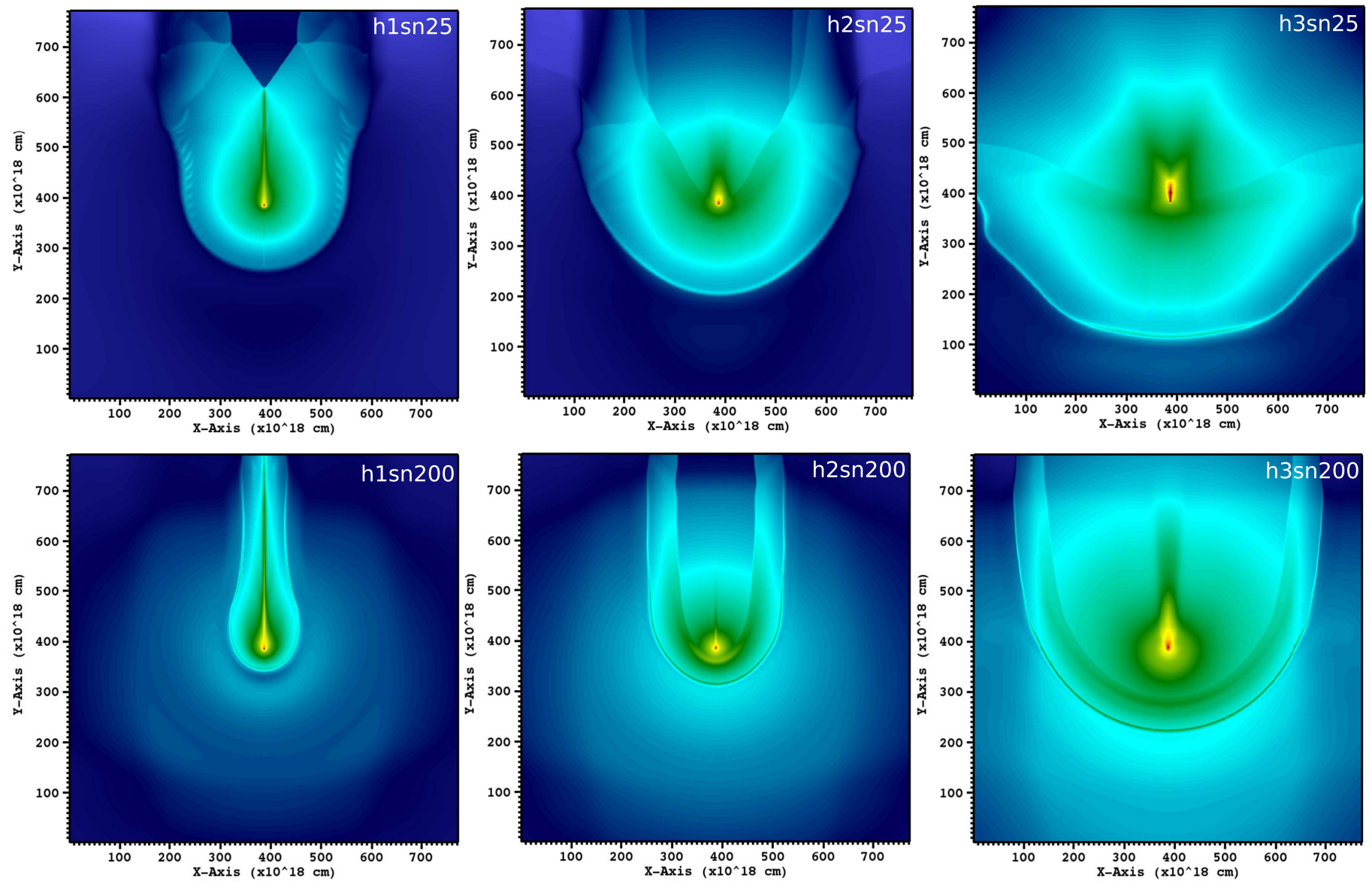

$\rho[\mathrm{g} / \mathrm{cc}]$

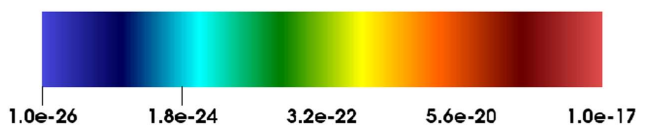

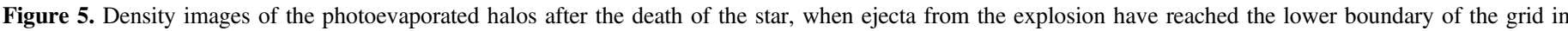

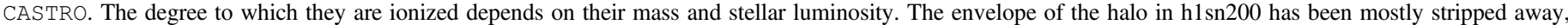
while the envelope of $\mathrm{h} 3 \mathrm{sn} 25$ is only slightly affected.

ionizes it on timescales of a few hundred kiloyears. This order of events may apply particularly to some of the sn 25 models in which metals do not come within $100 \mathrm{pc}$ of the core because of weaker UV fluxes and explosion energies. Our models therefore suggest that chemically enriched star formation in halos in close proximity to $\mathrm{SNe}$ should not be taken as a given, in contrast to what is usually assumed in semianalytical models and large-scale numerical simulations, since Pop III stars may form in them rather than Pop II stars in some cases.

\subsection{Distribution of Metals in the Halo}

We show mixing due to RT and $\mathrm{KH}$ instabilities on small scales in the h1sn200 model in Figure 8. Here, a fraction of the range of spatial scales on which mixing occurs is shown, with intricate structures with large surface areas that efficiently fold metals in with $\mathrm{H}$ and $\mathrm{He}$ gas on scales of 250, 80, 20, and $4 \mathrm{pc}$. To quantify mixing, radially averaged metallicities are plotted as a function of distance from the center of the halo for all six runs in Figure 9. Metals from the three PI SNe reach greater depths in the core and enrich it to higher metallicities than the three CC SNe because of their higher energies and much larger metal yields, $\sim 50 M_{\odot}$ versus a few $M_{\odot}$. The regions that are enriched by $\mathrm{CC} \mathrm{SNe}$ on average only have metallicities of $10^{-4}-10^{-5} Z_{\odot}$ and will only form Pop II stars if dust is present (e.g., Schneider et al. 2006; Klessen et al. 2012). It is therefore again not a given that Pop II stars will form, although some gas in the halo will be at metallicities above the spherical average and may form such stars. The disparity in mixing between PI and CC SNe is striking, with metallicities tapering off slowly at the center in the sn200 models but falling sharply at 70-100 pc in the sn 25 runs. Even in the core of the halo in the h1sn200 model the metallicity is $10^{-3}-10^{-4} Z_{\odot}$, enough to trigger the formation of a Pop II star. We define the mixing efficiency, $\epsilon(z)=M_{\text {met }}(z) / M_{\text {halo }}$, where $M_{\text {met }}(z)$ is the mass of metalenriched gas at a minimum metallicity $z$, and $M_{\text {halo }}$ is the mass of the photoevaporated halo. $\epsilon$ is sensitive to $z$ and $\mathrm{SN}$ type. For $z=10^{-5}, \epsilon$ of a CC SN and a PI SN are about $(2-5) \times 10^{-3}$ and $(3-6) \times 10^{-2}$, respectively. We list $\epsilon(z)$ values for each model in Table 2.

\subsection{Mixing in 3D}

The development of turbulent cascades due to RT and $\mathrm{KH}$ instabilities is different in 3D and 2D, and it might be expected that mixing is more efficient in $3 \mathrm{D}$ because of the greater 

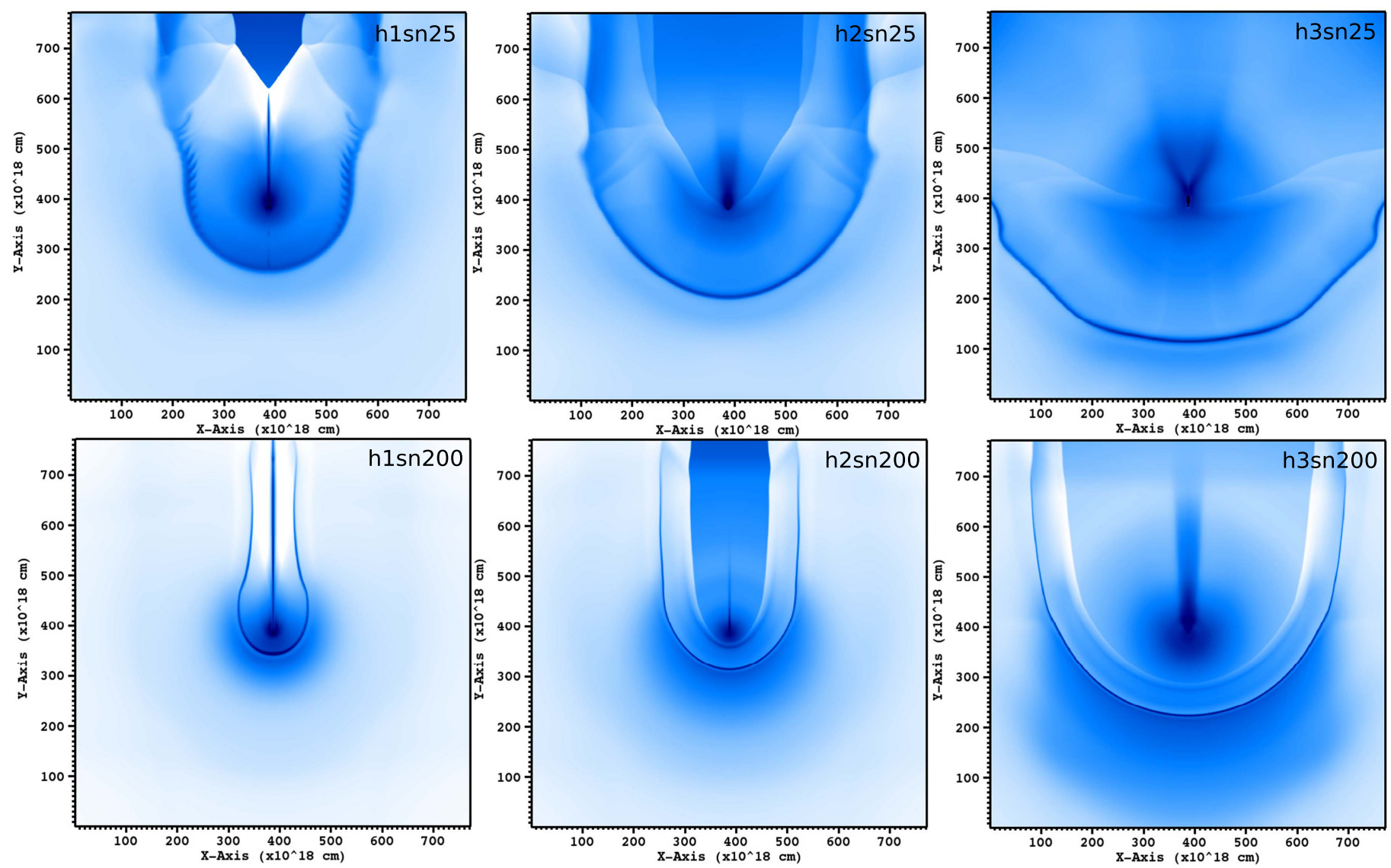

$\mathrm{T}[\mathrm{K}]$

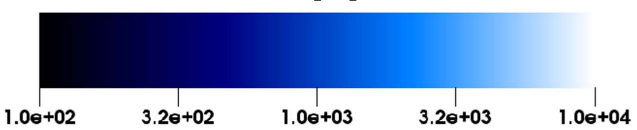

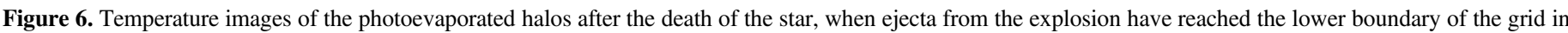

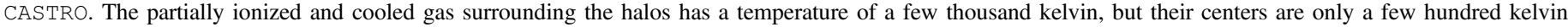
because they are shielded from stellar radiation, so $\mathrm{H}_{2}$ cooling can occur.

number of degrees of freedom of the flow. For example, the topology of I-front instabilities is quite different in $3 \mathrm{D}$, and they tend to devolve into turbulent flows much more efficiently (Whalen \& Norman 2008a, 2008b). Here, we run the h1 1 sn200 model in 3D to determine whether mixing is enhanced or suppressed. To set up this model, we rotate the $2 \mathrm{D}$ profile of the halo and its ionized envelope around its axis of symmetry and align and center it along the $z$-axis of a 3D Cartesian box in CASTRO. Had I-front instabilities developed in the $\mathrm{H}$ II region around the halo, it would have been necessary to rerun the ZEUS-MP model in 3D as well because the structure of the instabilities would have been different, but none were present in this model. The simulation box is $512^{3}$, with lower and upper boundaries at 0 and $250 \mathrm{pc}$ along all three axes. Metals flow in at the $z=0$ boundary, and outflow BCs are set on the other five boundaries. Two levels of AMR refinement $\left(2^{2}\right)$ in each dimension are used to achieve an effective resolution of $2048^{3}$, or $0.122 \mathrm{pc}$. The run required 1.5 million CPU hours on 6000 cores on the Cray XC30 machine Aterui at CfCA at NAOJ.

We show densities and metallicities for the halo at $10 \mathrm{Myr}$ in Figure 10. Both images reveal significant turbulent mixing, and metals again reach the core of the halo. Azimuthal averages of the metallicity in the 3D run are plotted with those from the 2D h1sn200 run in Figure 9. The bulk kinetic energy of the flow is more efficiently dissipated by the additional degree of freedom in the turbulence in $3 \mathrm{D}$, which enhances mixing at intermediate depths in the halo but results in fewer metals reaching the core, which is enriched to $\sim 10^{-5} Z_{\odot}$ instead of a few times $10^{-4} Z_{\odot}$. However, if dust forms in the metals, it will still trigger the formation of Pop II stars there.

\subsection{Comparison to Previous Models}

In contrast to Cen \& Riquelme (2008), we find that metals from $\mathrm{SNe}$ can mix well with primordial halos when the evaporation of the halo by the progenitor star is taken into account because ionizing UV from the star peels away the outer layers of the halo, partially exposing its core, and the outer layers of the halo are blown back toward the star, collide with debris from the explosion, and mix efficiently with it. Ejecta from the SN could only mix with the outer regions of the halo in Cen \& Riquelme (2008) because they are not stripped away by the radiation, so only a few percent of its mass was chemically enriched (by KH shear instabilities in its outermost layers). Preprocessing the halo with UV radiation from the star 

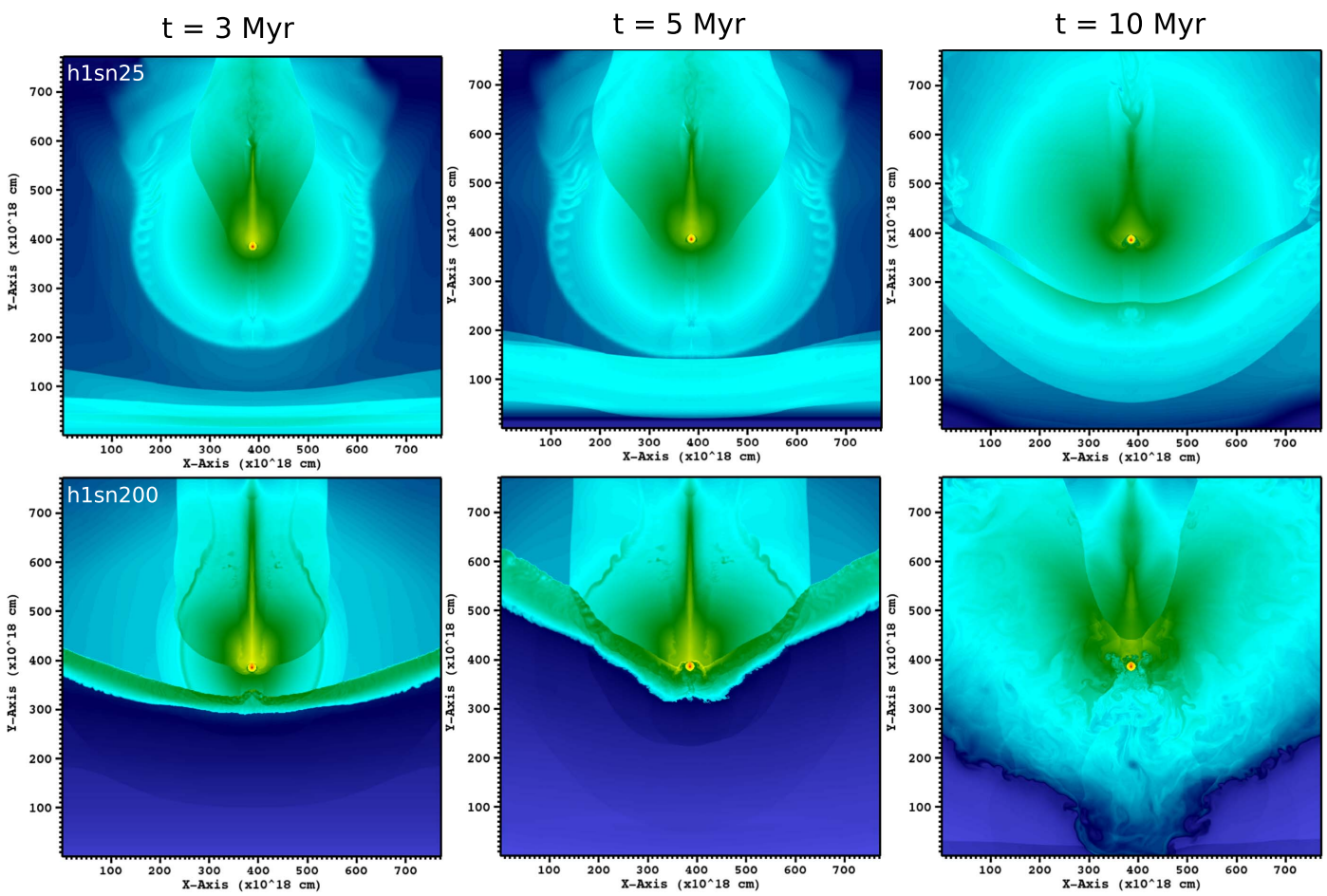

\section{$\rho[\mathrm{g} / \mathrm{cc}]$}
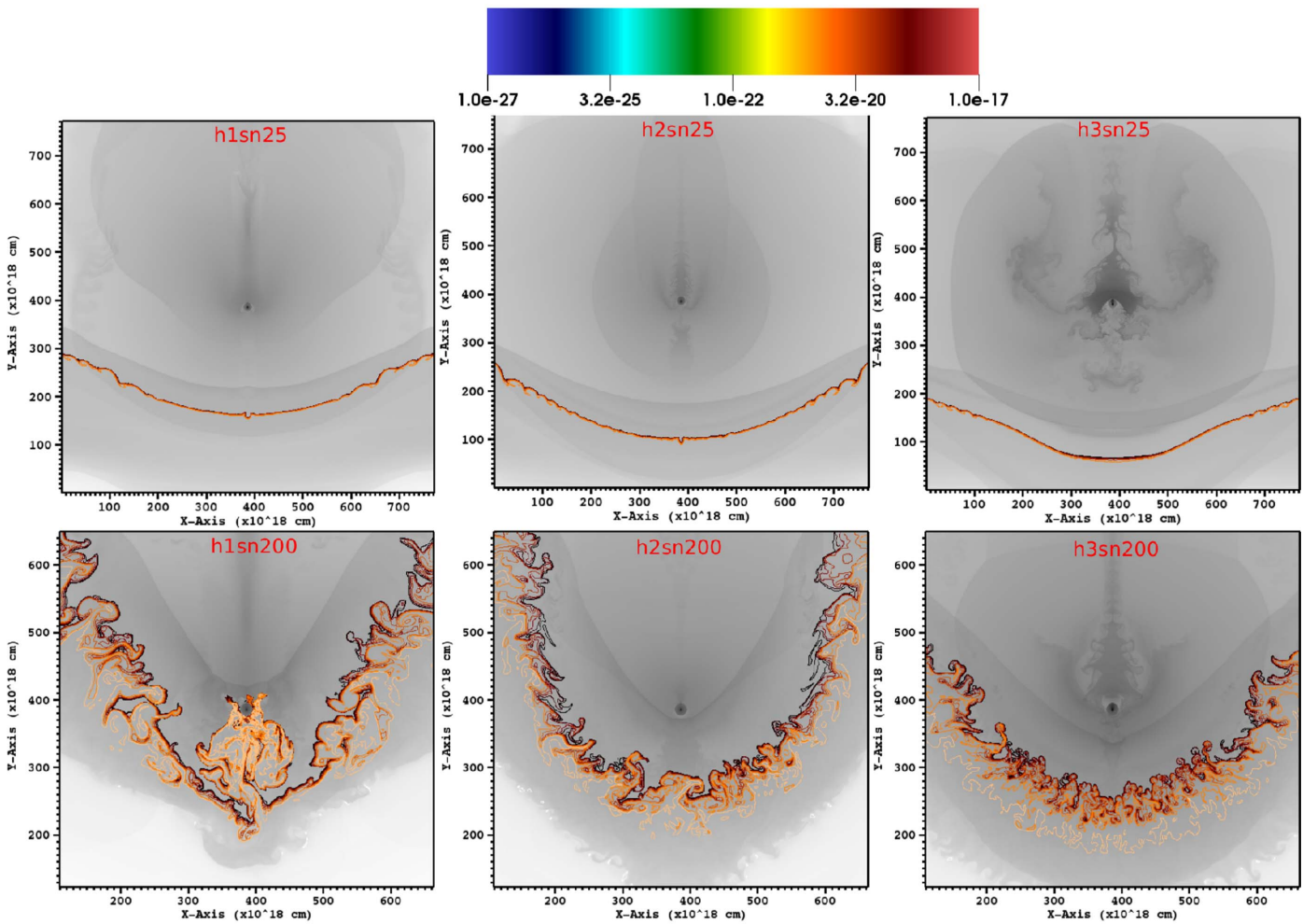

$\rho[g / c c]$

$\mathrm{Z}[\mathrm{Z} \odot]$
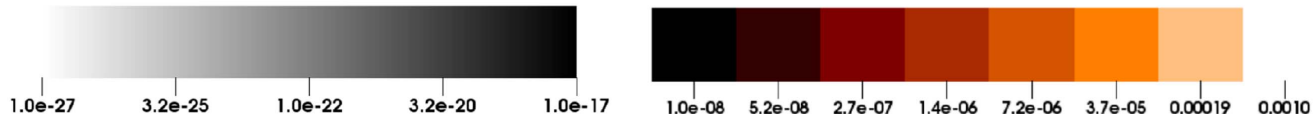

Figure 7. Upper panels: collision of the SN with the halo at 3, 5, and $10 \mathrm{Myr}$. In the $25 M_{\odot}$ runs the shock stalls 50 pc from the center of the halo but reaches and disrupts the center in the $200 M_{\odot}$ models. Bottom panels: distribution of metals in the halo at 10 Myr. Metals are halted $70-100 \mathrm{pc}$ from the center of the halo in the $25 M_{\odot}$ runs because of lower UV fluxes and explosion energies, so Pop II stars are less likely to form in the core but may occur in an arc around it. The $200 M_{\odot}$ SN has a more violent impact with the halo, and more prominent fluid instabilities appear. Mixing enables metals to reach the core of the halo in the h1sn200 run. 

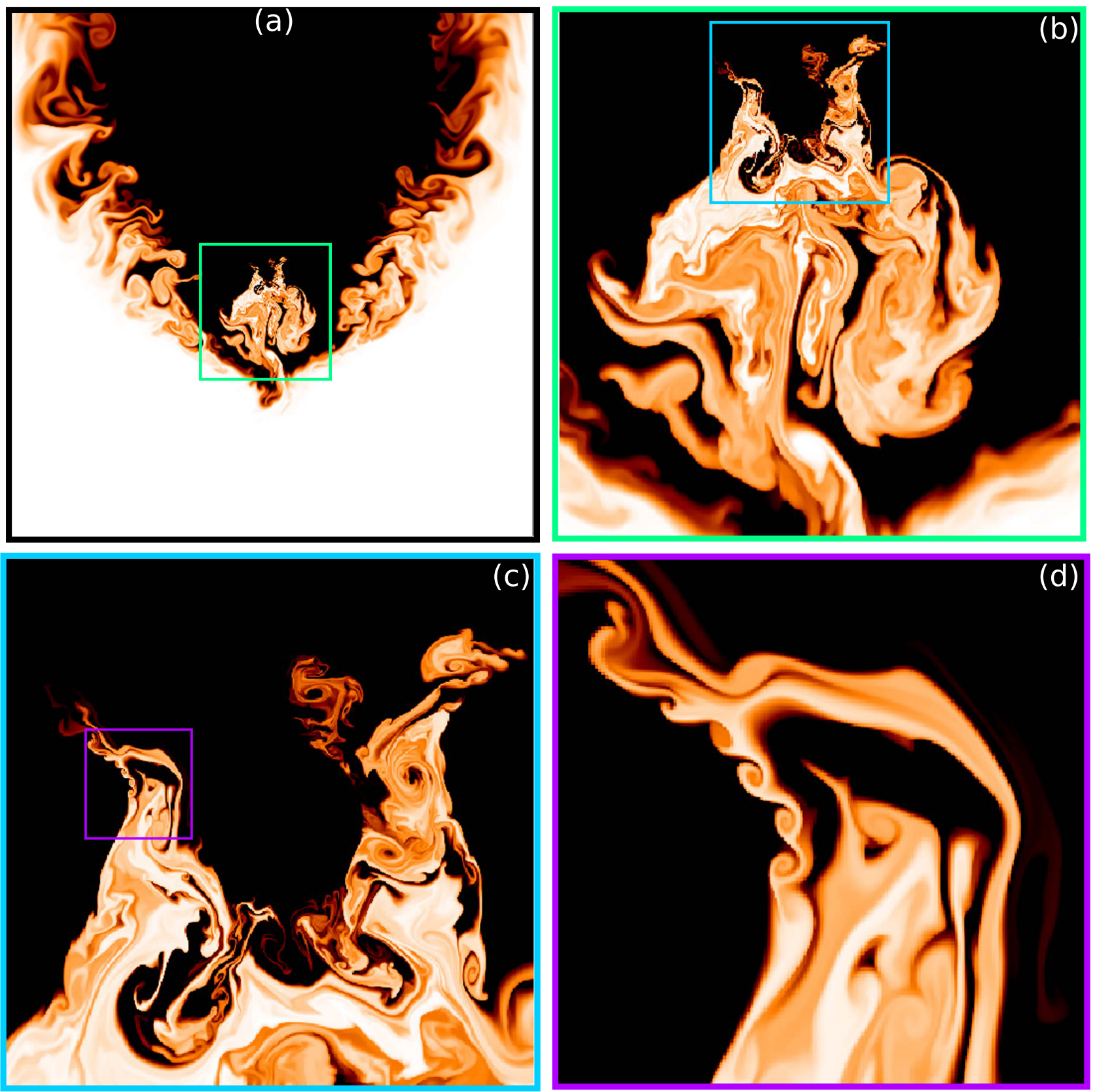

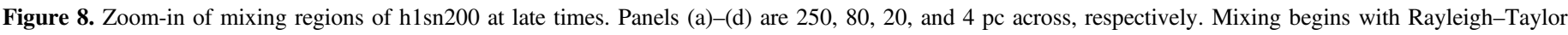
instabilities that develop into Kelvin-Helmholz shear instabilities that later cause the flow to become highly turbulent.

is therefore crucial to how metals from the $\mathrm{SN}$ later mix with the halo.

Smith et al. (2015) modeled the formation of a secondgeneration Pop II star in the debris of a Pop III SN in cosmological environments in the Enzo AMR code (Bryan et al. 2014). In their model, a $40 M_{\odot}$ Pop III star formed $200 \mathrm{pc}$ from a $3 \times 10^{5} M_{\odot}$ halo, partly evaporated it, and then exploded, with an energy of $10 \mathrm{~B}$ and a metal yield of $11.2 M_{\odot}$ (Nomoto et al. 2006). The SN crashes into the halo at $6 \mathrm{Myr}$ and enriches its core to $Z \sim 2 \times 10^{-5} Z_{\odot}$, forming a Pop II star via dust cooling. These results are consistent with our models because their initial UV fluxes and explosion energies were intermediate to those of our 25 and $200 M_{\odot}$ stars. Unlike our sn25 runs, metals from the $\mathrm{SN}$ in their simulation reached the core of the neighboring halo because it was exposed to higher UV fluxes over the life of the star (due to its larger mass and greater proximity to the halo) and because the explosion was more energetic and synthesized more metals. But the metallicity to which the core was enriched was smaller than in our sn 200 models because of the even greater UV flux, blast energy, and metal yield of the $200 M_{\odot}$ star.

Ritter et al. (2016) examined the low-energy explosion of a $60 M_{\odot}$ Pop III star inside a $\sim 10^{6} M_{\odot}$ halo that was not fully ionized by the star. A dense neutral clump in the halo persisted and was later overrun by metals from the SN. They found that the outer regions of the clump were only marginally enriched by metals, consistent with the mixing found in our $25 M_{\odot}$ SN models. From the resolution of the simulation it was not clear 


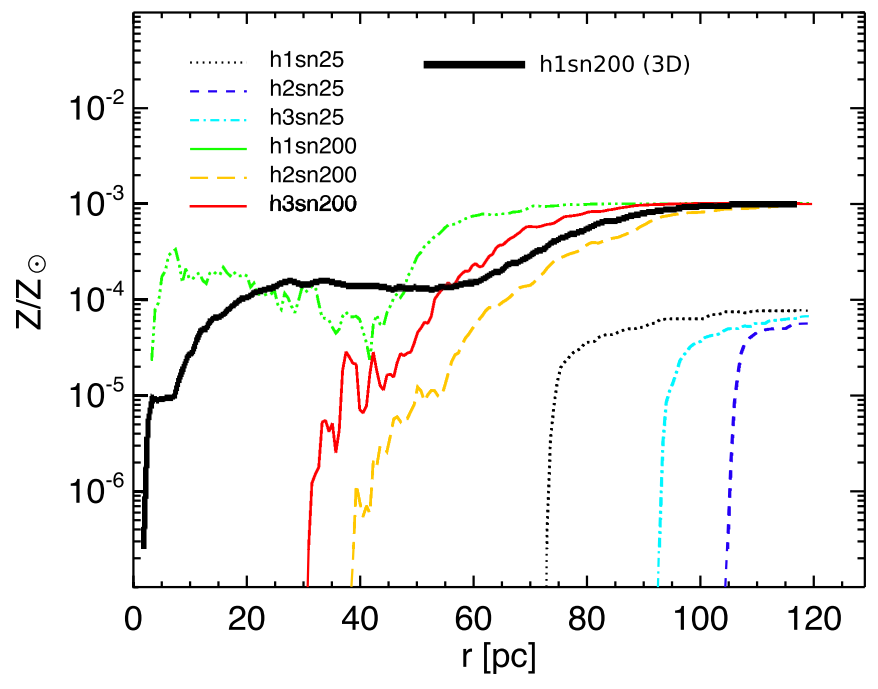

Figure 9. Spherically averaged metallicity profiles for the six 2D runs and the $3 \mathrm{D}$ h2sn 200 run at $10 \mathrm{Myr}$. Metals from the $25 M_{\odot}$ CC SN come no closer than $70-100 \mathrm{pc}$ to the core in all three halos but come much closer to the core in the $200 M_{\odot}$ runs, even reaching it in the h1sn200 run. In this run a Pop II star could form at the center of the halo. In the other five runs both Pop II stars may form at the dense relic $\mathrm{H}$ II region instead of the centers of halos. More metals reach the center of the halo in the h1sn200 model in $2 \mathrm{D}$ than in $3 \mathrm{D}$, due to the turbulent energy cascade.

Table 2

Mixing Efficiency $\epsilon(z)$

\begin{tabular}{lllcc}
\hline \hline Model & $10^{-3} Z_{\odot}$ & $10^{-4} Z_{\odot}$ & $10^{-5} Z_{\odot}$ & $10^{-6} Z_{\odot}$ \\
\hline h1sn25 & 0.00 & $7.07 \times 10^{-6}$ & $5.01 \times 10^{-3}$ & $5.06 \times 10^{-3}$ \\
h1sn200 & $1.80 \times 10^{-7}$ & $1.81 \times 10^{-2}$ & $2.69 \times 10^{-2}$ & $3.37 \times 10^{-2}$ \\
h2sn25 & 0.00 & 0.00 & $1.50 \times 10^{-4}$ & $1.53 \times 10^{-4}$ \\
h2sn200 & $3.32 \times 10^{-8}$ & $2.00 \times 10^{-3}$ & $4.29 \times 10^{-3}$ & $6.02 \times 10^{-3}$ \\
h3sn25 & 0.00 & $6.85 \times 10^{-6}$ & $2.29 \times 10^{-3}$ & $2.32 \times 10^{-3}$ \\
h3sn200 & $2.80 \times 10^{-6}$ & $2.53 \times 10^{-2}$ & $3.69 \times 10^{-2}$ & $4.61 \times 10^{-2}$ \\
\hline
\end{tabular}

Note. $\epsilon(z)=M_{\text {met }}(z) / M_{\text {halo }}$, where $M_{\text {met }}(z)$ is the mass of metal-enriched gas at a minimum metallicity $z$, and $M_{\text {halo }}$ is the mass of the photoevaporated halo.

whether this clump would collapse to a Pop III star or a Pop II star, but this model and the Smith et al. (2015) model exhibit the different degrees of mixing bracketed by our simulations.

\section{Discussion and Conclusion}

There is a region in the parameter space defined by the mass of the neighbor star, proximity to the halo, and the mass of the halo outside of which the SN cannot result in prompt Pop II star formation. If the star is too close, too much of the halo will be evaporated, and whatever gas remains will be stripped from the core by the ram pressure of the SN ejecta. The evaporative flow back toward the star will also be too diffuse to efficiently mix with the oncoming metals and form new stars. If the star is too far away, too little of the halo is blown off, and its core is mostly shielded from metals from the blast. Not enough mass flows back toward the star to mix efficiently or fragment into new stars. The range of parameters in which second-generation Pop II stars may form as a direct result of the blast remains to be surveyed.

Our models also indicate that even within this space the contamination of a halo with metals does not by itself guarantee the formation of Pop II stars, in contrast to what is usually assumed in semianalytical models or large-scale cosmological simulations of high-redshift star and galaxy formation. The key in each case are the relative timescales of metal cooling and fragmentation in enriched gas and $\mathrm{H}_{2}$ cooling in the pristine core because the formation of one type of star may preclude the formation of the other, depending on which happens first. More simulations of halo-SN pairs with the cooling physics are needed to resolve fragmentation and collapse. Those are very important to determine the branching ratios of Pop II and Pop III star formation (or both) in semianalytic models and simulations.

Although our halos are extracted from cosmological simulations, they are idealized in several respects. Real halos have complex 3D geometries and are threaded by cosmological filaments that are not present in our simulations. Collision of $\mathrm{SN}$ ejecta with the filaments could result in star formation in their outer layers, with some of these stars later being carried into the halo by the flow. Accretion from filaments and mergers with other structures also produce mild subsonic turbulence in the halo that could allow radiation from the star to reach to greater depths and promote mixing in the core (Smith et al. 2015; see also Greif et al. 2008). Our models exclude turbulence due to mergers or accretion but capture turbulence due to RT and $\mathrm{KH}$ instabilities during the collision, and at a higher resolution than is currently achieved in cosmological simulations. That said, because we ignore turbulence in the halo prior to the collision, our mixing results should be taken to be lower limits (the same is true of Cen \& Riquelme 2008, even without preprocessing of the halo with radiation). How precollision turbulence enhances Pop II star formation in the halo will be investigated in future models, which may also include a prescription for turbulent mixing on unresolved scales (Klessen \& Lin 2003; Greif et al. 2009).

As discussed earlier, molecular hydrogen cooling in the relic $\mathrm{H}$ II region plays a role in mixing because it generally causes the gas to collapse to higher densities. This effect is greatest in the earliest generations of stars because they build up a global LW background over time that dissociates $\mathrm{H}_{2}$ and slows down cooling in these regions. Reduced $\mathrm{H}_{2}$ mass fractions would result in puffier, more diffuse relic $\mathrm{H}$ II regions that mix less efficiently with SN ejecta and suppress the formation of enriched stars. Mixing and fragmentation in global LW backgrounds will be studied in future models.

Another axis in the parameter space of mixing that is beyond the scope of this study is the initial degree of collapse of the halo at the time of photoevaporation and collision with the ejecta. Central densities in our halos were $\sim 10^{5}-10^{6} \mathrm{~cm}^{-3}$, so collapse was in progress but no stars had formed. At even higher central densities the core of the halo is even more shielded from LW and ionizing UV and metals cannot as easily reach it. We therefore expect the contamination of the core to be reduced at later stages of collapse but not mixing at intermediate or outer layers. If the center of the halo rises to densities of $\gtrsim 10^{8} \mathrm{~cm}^{-3}$ before metals reach it, the core fully molecularizes and collapses to a Pop III star with little or no enrichment.

Because our simulations do not include cooling due to metals, we cannot say for certain when secondary enriched star formation will happen, only where it might occur as a result of mixing. Our models also lack cooling due to molecules and dust, which would also promote new star formation in mixed zones. Metals and molecules can cool gas down to the temperature of the CMB at densities of around $10^{4} \mathrm{~cm}^{-3}$. Dust cooling occurs at much higher densities, regimes where the characteristic mass scale of fragmentation is much smaller 

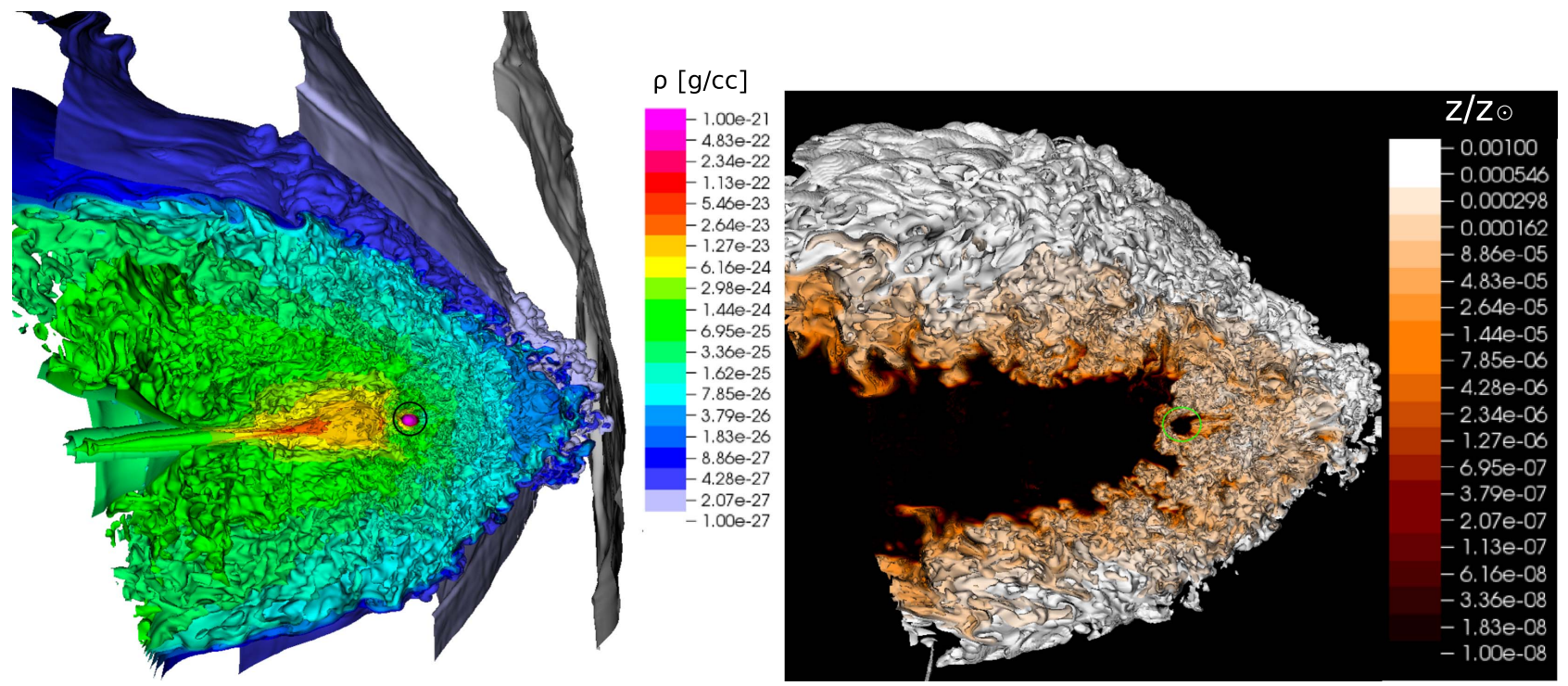

Figure 10. Densities (left) and metallicities (right) in the halo in the h1sn200 run in 3D. Colors show isosurfaces of density after mixing. Turbulent flows are visible everywhere in zones of chemical enrichment. The black circle marks the core of the halo, $r \sim 5 \mathrm{pc}$. Metals also reach the core in this $3 \mathrm{D}$ run.

owing to the $\rho^{-1 / 2}$ dependence of the Jeans mass on density (Schneider et al. 2006). Numerical models indicate that Pop III $\mathrm{SNe}$ can produce up to several solar masses of molecules and dust (Cherchneff \& Dwek 2009, 2010), but the amount of dust that survives passage through the reverse shock in the SN ejecta remains unclear (Bianchi \& Schneider 2007; Silvia et al. 2012). How early chemical enrichment schemes produce new stars will not be fully known until cooling due to all three species is implemented in simulations, which is now under development.

But cooling could also influence mixing prior to fragmentation by radiating away some of the bulk kinetic energy of the flow. It is clear from the difference in degree of mixing between PI SNe and less-energetic CC SNe that the loss of this energy could dampen mixing and reduce the depths to which metals permeate the halo. However, the mixing in our models is consistent with that in cosmological simulations that do include these cooling processes, so it is unlikely that their exclusion substantially alters our results.

A few dwarf galaxies have now been identified as candidates for "one-shot" chemical enrichment, being contaminated by metals from just one Pop III SN at an early epoch and mostly isolated from mergers and accretion flows thereafter (Frebel \& Bromm 2012). The elemental abundances and metallicity of Leo IV, for example, are a good fit to the yield of a single $10 M_{\odot}$ Pop III CC SN (Simon et al. 2010). But our simulations suggest that Leo IV could not have been enriched by an external explosion of this mass and energy. The UV flux from the progenitor would not have evaporated much of the halo prior to the explosion, and the energy of the blast would not have been sufficient to drive efficient mixing afterward (besides the fact that only metals in the solid angle subtended by the halo could have mixed with it). It is more likely that the elements in Leo IV came from internal enrichment, in which the weak UV flux of the Pop III star failed to ionize its host halo and the star exploded in a relatively compact and highdensity H II region. This scenario would lead to violent mixing, with few of the metals escaping the halo (Whalen et al. 2008c; Ritter et al. 2012).
Finally, we have only considered one scenario in which metals from an $\mathrm{SN}$ enrich gas and trigger new star formation. Many others can be imagined, such as collision of SN ejecta with the dense shell of a nearby relic $\mathrm{H}$ II region or with another SN remnant. Even a single expanding SN front can crumple owing to overstabilities and fragment, perhaps forming new stars. These and many other channels of chemically enriched star formation are yet to be investigated.

The authors are grateful to the anonymous referee for providing insightful comments. We would like to thank Mike Norman and Volker Bromm for useful discussions on the science in this paper and Ann Almgren, Mike Zingale, and Weiqun Zhang for their technical support with CASTRO. K.C. acknowledges the support of the EACOA Fellowship of the East Asian Core Observatories Association and the hospitality of the Aspen Center for Physics, which is supported by National Science Foundation grant PHY-1066293. Work done at UCSC was supported by an IAU-Gruber Fellowship, the DOE HEP Program (DE-SC0010676), and the NASA Theory Program (NNX14AH34G). D.J.W. was supported by STFC New Applicant Grant ST/P000509/1, and K.M.J.W. and D.J. W. were supported by the European Research Council under the European Community's Seventh Framework Programme (FP7/2007-2013) via the ERC Advanced Grant "STARLIGHT: Formation of the First Stars" (project no. 339177). All CASTRO simulations were performed at NERSC and the CfCA at NAOJ.

\section{References}

Abel, T., Wise, J. H., \& Bryan, G. L. 2007, ApJL, 659, L87 Agarwal, B., Khochfar, S., Johnson, J. L., et al. 2012, MNRAS, 425, 2854 Almgren, A. S., Beckner, V. E., Bell, J. B., et al. 2010, ApJ, 715, 1221 Alvarez, M. A., Bromm, V., \& Shapiro, P. R. 2006, ApJ, 639, 621 Alvarez, M. A., Wise, J. H., \& Abel, T. 2009, ApJL, 701, L133 Aoki, W., Suda, T., Beers, T. C., \& Honda, S. 2015, AJ, 149, 39 Aoki, W., Tominaga, N., Beers, T. C., Honda, S., \& Lee, Y. S. 2014, Sci, 345,912

Beers, T. C., \& Christlieb, N. 2005, ARA\&A, 43, 531

Bianchi, S., \& Schneider, R. 2007, MNRAS, 378, 973

Bonifacio, P., Caffau, E., Spite, M., et al. 2015, A\&A, 579, A28 
Bromm, V., Ferrara, A., Coppi, P. S., \& Larson, R. B. 2001, MNRAS, 328, 969

Bryan, G. L., Norman, M. L., O’Shea, B. W., et al. 2014, ApJS, 211, 19

Caffau, E., Bonifacio, P., François, P., et al. 2012, A\&A, 542, A51

Caffau, E., Bonifacio, P., Sbordone, L., et al. 2013, A\&A, 560, A71

Cayrel, R., Depagne, E., Spite, M., et al. 2004, A\&A, 416, 1117

Cen, R., \& Riquelme, M. A. 2008, ApJ, 674, 644

Chen, K.-J., Bromm, V., Heger, A., Jeon, M., \& Woosley, S. 2015, ApJ, 802,13

Chen, K.-J., Heger, A., Whalen, D. J., et al. 2017, MNRAS, 467, 4731

Chen, K.-J., Heger, A., Woosley, S., Almgren, A., \& Whalen, D. J. 2014a, ApJ, 792, 44

Chen, K.-J., Heger, A., Woosley, S., et al. 2014b, ApJ, 790, 162

Chen, K.-J., Woosley, S., Heger, A., Almgren, A., \& Whalen, D. J. 2014c, ApJ, 792, 28

Cherchneff, I., \& Dwek, E. 2009, ApJ, 703, 642

Cherchneff, I., \& Dwek, E. 2010, ApJ, 713, 1

Chiaki, G., Yoshida, N., \& Kitayama, T. 2013, ApJ, 762, 50

Choi, J.-H., Shlosman, I., \& Begelman, M. C. 2013, ApJ, 774, 149

Clark, P. C., Glover, S. C. O., \& Klessen, R. S. 2008, ApJ, 672, 757

Cooke, R., Pettini, M., Steidel, C. C., Rudie, G. C., \& Jorgenson, R. A. 2011, MNRAS, 412, 1047

Cooke, R. J., \& Madau, P. 2014, ApJ, 791, 116

Cooke, R. J., Pettini, M., \& Jorgenson, R. A. 2015, ApJ, 800, 12

de Souza, R. S., Ishida, E. E. O., Johnson, J. L., Whalen, D. J., \& Mesinger, A. 2013, MNRAS, 436, 1555

de Souza, R. S., Ishida, E. E. O., Whalen, D. J., Johnson, J. L., \& Ferrara, A. 2014, MNRAS, 442, 1640

Dopcke, G., Glover, S. C. O., Clark, P. C., \& Klessen, R. S. 2011, ApJL, 729, L3

Frebel, A. 2010, AN, 331, 474

Frebel, A., Aoki, W., Christlieb, N., et al. 2005, Natur, 434, 871

Frebel, A., \& Bromm, V. 2012, ApJ, 759, 115

Glover, S. C. O., \& Abel, T. 2008, MNRAS, 388, 1627

Gnedin, N. Y., \& Hollon, N. 2012, ApJS, 202, 13

Gray, W. J., \& Scannapieco, E. 2011, ApJ, 733, 88

Greif, T. H., Glover, S. C. O., Bromm, V., \& Klessen, R. S. 2009, MNRAS, 392, 1381

Greif, T. H., Glover, S. C. O., Bromm, V., \& Klessen, R. S. 2010, ApJ, 716,510

Greif, T. H., Johnson, J. L., Klessen, R. S., \& Bromm, V. 2008, MNRAS, 387,1021

Haemmerlé, L., Woods, T. E., Klessen, R. S., Heger, A., \& Whalen, D. J. 2017, arXiv: 1705.09301

Hartwig, T., Bromm, V., Klessen, R. S., \& Glover, S. C. O. 2015, MNRAS, 447,3892

Hasegawa, K., Umemura, M., \& Susa, H. 2009, MNRAS, 395, 1280

Heger, A., \& Woosley, S. E. 2002, ApJ, 567, 532

Iliev, I. T., Shapiro, P. R., \& Raga, A. C. 2005, MNRAS, 361, 405

Iwamoto, N., Umeda, H., Tominaga, N., Nomoto, K., \& Maeda, K. 2005, Sci, 309,451

Jappsen, A.-K., Mac Low, M.-M., Glover, S. C. O., Klessen, R. S., \& Kitsionas, S. 2009, ApJ, 694, 1161

Jeon, M., Pawlik, A. H., Greif, T. H., et al. 2012, ApJ, 754, 34

Joggerst, C. C., Almgren, A., Bell, J., et al. 2010, ApJ, 709, 11

Joggerst, C. C., \& Whalen, D. J. 2011, ApJ, 728, 129

Johnson, J. L., Greif, T. H., Bromm, V., Klessen, R. S., \& Ippolito, J. 2009 MNRAS, 399, 37

Johnson, J. L., Whalen, D. J., Agarwal, B., Paardekooper, J.-P., \& Khochfar, S. 2014, MNRAS, 445, 686

Karlsson, T., Johnson, J. L., \& Bromm, V. 2008, ApJ, 679, 6

Kasen, D., Woosley, S. E., \& Heger, A. 2011, ApJ, 734, 102

Keller, S. C., Bessell, M. S., Frebel, A., et al. 2014, Natur, 506, 463

Kitayama, T., \& Yoshida, N. 2005, ApJ, 630, 675

Kitayama, T., Yoshida, N., Susa, H., \& Umemura, M. 2004, ApJ, 613, 631

Klessen, R. S., Glover, S. C. O., \& Clark, P. C. 2012, MNRAS, 421, 3217

Klessen, R. S., \& Lin, D. N. 2003, PhRvE, 67, 046311

Lai, D. K., Bolte, M., Johnson, J. A., et al. 2008, ApJ, 681, 1524

Latif, M. A., Schleicher, D. R. G., Schmidt, W., \& Niemeyer, J. 2013a MNRAS, 430, 588

Latif, M. A., Schleicher, D. R. G., Schmidt, W., \& Niemeyer, J. 2013b MNRAS, 433, 1607

Mackey, J., Bromm, V., \& Hernquist, L. 2003, ApJ, 586, 1

Magg, M., Hartwig, T., Glover, S. C. O., Klessen, R. S., \& Whalen, D. J. 2016, MNRAS, 462, 3591

Meiksin, A., \& Whalen, D. J. 2013, MNRAS, 430, 2854
Mesler, R. A., Whalen, D. J., Lloyd-Ronning, N. M., Fryer, C. L., \& Pihlström, Y. M. 2012, ApJ, 757, 117

Mesler, R. A., Whalen, D. J., Smidt, J., et al. 2014, ApJ, 787, 91

Nomoto, K., Tominaga, N., Umeda, H., Kobayashi, C., \& Maeda, K. 2006 NuPhA, 777, 424

Norris, J. E., Yong, D., Bessell, M. S., et al. 2013, ApJ, 762, 28

O'Shea, B. W., Abel, T., Whalen, D., \& Norman, M. L. 2005, ApJL, 628, L5

O'Shea, B. W., Wise, J. H., Xu, H., \& Norman, M. L. 2015, ApJL, 807, L12

Park, K., \& Ricotti, M. 2011, ApJ, 739, 2

Park, K., \& Ricotti, M. 2012, ApJ, 747, 9

Park, K., \& Ricotti, M. 2013, ApJ, 767, 163

Pawlik, A. H., Milosavljević, M., \& Bromm, V. 2011, ApJ, 731, 54

Pawlik, A. H., Milosavljević, M., \& Bromm, V. 2013, ApJ, 767, 59

Richardson, M. L. A., Scannapieco, E., \& Gray, W. J. 2013, ApJ, 778, 80

Ricotti, M., Gnedin, N. Y., \& Shull, J. M. 2001, ApJ, 560, 580

Ritter, J. S., Safranek-Shrader, C., Gnat, O., Milosavljević, M., \& Bromm, V. 2012, ApJ, 761, 56

Ritter, J. S., Safranek-Shrader, C., Milosavljević, M., \& Bromm, V. 2016, MNRAS, 463, 3354

Safranek-Shrader, C., Milosavljević, M., \& Bromm, V. 2014, MNRAS, 438, 1669

Schaerer, D. 2002, A\&A, 382, 28

Schauer, A. T. P., Agarwal, B., Glover, S. C. O., et al. 2017, MNRAS, 467, 2288

Schauer, A. T. P., Whalen, D. J., Glover, S. C. O., \& Klessen, R. S. 2015, MNRAS, 454, 2441

Schleicher, D. R. G., Palla, F., Ferrara, A., Galli, D., \& Latif, M. 2013, A\&A, 558, A59

Schneider, R., Ferrara, A., Natarajan, P., \& Omukai, K. 2002, ApJ, 571, 30

Schneider, R., Omukai, K., Inoue, A. K., \& Ferrara, A. 2006, MNRAS, 369, 1437

Shapiro, P. R., Iliev, I. T., \& Raga, A. C. 2004, MNRAS, 348, 753

Silvia, D. W., Smith, B. D., \& Shull, J. M. 2012, ApJ, 748, 12

Simon, J. D., Frebel, A., McWilliam, A., Kirby, E. N., \& Thompson, I. B 2010, ApJ, 716, 446

Smidt, J., Whalen, D. J., Chatzopoulos, E., et al. 2015, ApJ, 805, 44

Smidt, J., Whalen, D. J., Johnson, J. L., \& Li, H. 2017, arXiv:1703.00449

Smidt, J., Whalen, D. J., Wiggins, B. K., et al. 2014, ApJ, 797, 97

Smith, B. D., \& Sigurdsson, S. 2007, ApJL, 661, L5

Smith, B. D., Turk, M. J., Sigurdsson, S., O’Shea, B. W., \& Norman, M. L. 2009, ApJ, 691, 441

Smith, B. D., Wise, J. H., O’Shea, B. W., Norman, M. L., \& Khochfar, S. 2015, MNRAS, 452, 2822

Susa, H. 2007, ApJ, 659, 908

Susa, H., \& Umemura, M. 2006, ApJL, 645, L93

Susa, H., Umemura, M., \& Hasegawa, K. 2009, ApJ, 702, 480

Tanaka, T., \& Haiman, Z. 2009, ApJ, 696, 1798

Truelove, J. K., \& McKee, C. F. 1999, ApJS, 120, 299

Volonteri, M. 2012, Sci, 337, 544

Whalen, D., Abel, T., \& Norman, M. L. 2004, ApJ, 610, 14

Whalen, D., Hueckstaedt, R. M., \& McConkie, T. O. 2010, ApJ, 712, 101

Whalen, D., \& Norman, M. L. 2006, ApJS, 162, 281

Whalen, D., \& Norman, M. L. 2008a ApJ, 673, 664

Whalen, D., O’Shea, B. W., Smidt, J., \& Norman, M. L. 2008a, ApJ, 679, 925

Whalen, D., Prochaska, J. X., Heger, A., \& Tumlinson, J. 2008b, ApJ, 682,1114

Whalen, D., van Veelen, B., O'Shea, B. W., \& Norman, M. L. 2008c, ApJ, 682,49

Whalen, D. J., Even, W., Smidt, J., et al. 2013a, ApJ, 778, 17

Whalen, D. J., \& Fryer, C. L. 2012, ApJL, 756, L19

Whalen, D. J., Fryer, C. L., Holz, D. E., et al. 2013b, ApJL, 762, L6

Whalen, D. J., Joggerst, C. C., Fryer, C. L., et al. 2013c, ApJ, 768, 95

Whalen, D. J., Johnson, J. L., Smidt, J., et al. 2013d, ApJ, 777, 99

Whalen, D. J., Johnson, J. L., Smidt, J., et al. 2013e, ApJ, 774, 64

Whalen, D. J., \& Norman, M. L. 2008b, ApJ, 672, 287

Whalen, D. J., Smidt, J., Even, W., et al. 2014a, ApJ, 781, 106

Whalen, D. J., Smidt, J., Heger, A., et al. 2014b, ApJ, 797, 9

Whalen, D. J., Smidt, J., Johnson, J. L., et al. 2013f, arXiv:1312.6330

Williams, R. J. R. 2002, MNRAS, 331, 693

Wise, J. H., Turk, M. J., Norman, M. L., \& Abel, T. 2012, ApJ, 745, 50

Woods, T. E., Heger, A., Whalen, D. J., Haemmerle, L., \& Klessen, R. S. 2017 arXiv: 1703.07480

Woodward, P., \& Colella, P. 1984, JCoPh, 54, 115

Yoshida, N., Oh, S. P., Kitayama, T., \& Hernquist, L. 2007a, ApJ, 663, 687

Yoshida, N., Omukai, K., \& Hernquist, L. 2007b, ApJL, 667, L117

Zhang, W., Howell, L., Almgren, A., Burrows, A., \& Bell, J. 2011, ApJS, 196,20 